\title{
Técnicas estatísticas para análise da qualidade da água em áreas irrigadas no baixo Rio São Francisco
}

Conhecer os impactos da agricultura irrigada na qualidade da água é muito importante quando se busca construir estratégias de gestão para revitalizar mananciais. O objetivo do estudo foi realizar o monitoramento físico-químico e analisar os possíveis impactos da agricultura irrigada na qualidade da água no baixo rio São Francisco, nos municípios Canindé do São Francisco (semiárido) e Neópolis (semiúmido) no de período de 03/2013 a 12/2014. Foram monitorados mensalmente vinte e dois parâmetros físico-químicos em quatros mananciais: rio da Onça, rio Betume, drenagem Betume e rio São Francisco. Os dados obtidos foram analisados se utilizando técnicas estáticas multivariadas para determinar suas inter-relações, como também, simplificar sua interpretação. Os parâmetros da qualidade da água estão relacionados principalmente com o conteúdo mineral, nutricional, matéria orgânica e grau de poluição. Dentre as regiões estudadas, o perímetro irrigado Califórnia apresentou um maior grau de eutrofização explicado principalmente pelos elevados valores de oxigênio dissolvido (OD), fósforo, nitrogênio e demanda bioquímica de oxigênio (DBO). O uso de técnicas estatísticos para analisar os resultados dos parâmetros físico-químicos, pode evidenciar os diversos problemas existentes nos perímetros irrigados no baixo rio São Francisco quanto ao manejo agrícola e a qualidade da água dos mananciais.

\section{Statistical techniques for water quality analysis in irrigated areas in the low São Francisco River}

\begin{abstract}
Knowing the impacts of irrigated agriculture on water quality is very important when trying to build management strategies to revitalize watercourses. The objective of the study was to perform physical-chemical monitoring and to analyze the possible impacts of irrigated agriculture on water quality in the lower São Francisco river, in the municipalities Canindé do São Francisco (semi-arid) and Neópolis (semi-humid) in the period of 03/2013 to 12/2014. Twenty-two physico-chemical parameters were monitored monthly in four watercourses: the Onça River, the Betume River, the Betume drainage and the São Francisco River. The data obtained were analyzed using multivariate static techniques to determine their interrelationships, as well as to simplify their interpretation. The parameters of water quality are mainly related to the mineral content, nutritional, organic matter and degree of pollution. Among the studied regions, the irrigated perimeter California presented a higher degree of eutrophication explained mainly by high values of dissolved oxygen (DO), phosphorus, nitrogen and biochemical oxygen demand (BOD). The use of statistical techniques to analyze the results of the physicochemical parameters can show various problems in the irrigated perimeters in the lower São Francisco River, regarding the agricultural management and the water quality of the springs.
\end{abstract}

Keywords: Environmental Monitoring; Physical-Chemical Parameters; Multivariate Analysis.

Topic: Engenharia Ambiental

Reviewed anonymously in the process of blind peer.
Received: 02/02/2020

Approved: 04/03/2020
Fábio Brandão Britto (iD)

Instituto Federal de Sergipe, Brasil

http://lattes.cnpq.br/3575178384246106

http://orcid.org/0000-0002-3830-7468

bradaobritto@gmail.com

Anderson Nascimento do Vasco (iD

Instituto Federal de Sergipe, Brasil

http://lattes.cnpq.br/7147160524840953

http://orcid.org/0000-0002-7031-3745

anderovasco@yahoo.com.br

Gilsia Fabiane Oliveira Morais (iD)

Instituto Federal de Sergipe, Brasi

http://lattes.cnpq.br/8285979094812095

http://orcid.org/0000-0001-9371-6339

gilsia.fabiane@ifs.edu.br
Antenor de Oliveira Aguiar Netto (iD

Universidade Federal de Sergipe, Brasil

http://lattes.cnpq.br/9527039294611376

http://orcid.org/0000-0002-3555-6776

antenor.ufs@gmail.com
Referencing this:

BRITTO, F. B.; VASCO, A. N.; MORAIS, G. F. O.; AGUIAR NETTO, A. O.. Técnicas estatísticas para análise da qualidade da água em áreas irrigadas no baixo Rio São Francisco. Revista Ibero Americana de Ciências Ambientais, v.11, n.2, p.192-203, 2020. DOI:

http://doi.org/10.6008/CBPC2179-6858.2020.002.0021 


\section{INTRODUÇÃO}

A gestão bem-sucedida dos recursos hídricos disponíveis numa bacia hidrográfica requer informações confiáveis sendo importante implementar programas de monitoramento para analisar qualidade da água dos mananciais por meio das características físicas, químicas ou biológicas. O monitoramento de mananciais por longos períodos e em várias estações de amostragem na maioria das vezes produz um banco de dados grande e complexo que abrange diversos tipos de parâmetros de qualidade da água. Essa complexidade dificulta a análise, a interpretação dos dados e a extração de informações úteis para a gestão adequada da qualidade da água, sendo o banco de dados muitas vezes subutilizado (TRINDADE et al., 2017).

O uso de métodos estatísticos é uma ferramenta de estudo, que pode ser utilizada na identificação e interpretação de situações de impactos ambientais na qualidade da água, uma vez que, é possível sintetizar um grande volume de informações complexas e conhecer os parâmetros mais importantes e suas interrelações na avaliação da qualidade da água.

Métodos estatísticos multivariados são excelentes ferramentas exploratórias para a interpretação desse complexo conjunto de informações e possibilitam a avaliação de longas séries de dados, sendo úteis para o acompanhamento e a predição da evolução da qualidade da água. Como também permitem compreender a influência que fatores como o uso e a ocupação do solo exercem sobre a qualidade das águas, oportunizando a identificação dos trechos mais críticos dos mananciais (GIRÃO et al., 2007; KUMARESAN et al., 2008; SOJKA et al., 2008; SCHAGERL et al., 2010; SONG et al., 2011; TRINDADE et al., 2017).

Ao longo do processo histórico de ocupação humana na região do baixo rio São Francisco sergipano, as atividades antrópicas promoveram mudanças significativas na dinâmica do rio (FERREIRA et al., 2011). Este fato, chama atenção da necessidade de fazer o monitoramento desses mananciais e encontrar respostas sobre os parâmetros de qualidade das águas que pode ter sido influenciada pelas atividades de usos múltiplos. Outro fator observado nos mananciais do rio São Francisco, é a criação de Perímetros irrigados em suas margens sem avaliar os possíveis impactos ambientais nos recursos hídricos.

Além disso, a distribuição de água em áreas irrigadas influencia diretamente o manejo, a qualidade, a eficiência e o custo da irrigação, assim como o desempenho da cultura no campo. $O$ excesso de água no solo pode provocar a lixiviação de nutrientes e contaminantes agrícolas, a redução na concentração de oxigênio disponível para as raízes e assim aumentar a incidência de pragas e doenças que contribui e estimula o uso de agrotóxico nas regiões dos perímetros irrigados (BARROS et al., 2008).

As péssimas condições físicas e econômicas dos perímetros irrigados em Sergipe, associada à utilização de métodos de irrigação inadequados, têm levado a sérios desafios à gestão adequada desses projetos de irrigação (HOLANDA et al., 2008). Outro fator é a falta do manejo dos recursos hídricos adequados sem monitoramento da qualidade da água, que pode estar contribuindo para o agravamento das condições ambientais destas regiões. Desta forma este estudo tem como objetivo fazer o monitoramento e análise dos parâmetros físico-químicos da água utilizando o método estatístico de Análise Multivariada e avaliar os 
impactos do perímetro irrigado no baixo rio São Francisco.

\section{MATERIAIS E MÉTODOS}

\section{Área de estudo}

A região do baixo São Francisco ocupa uma área de $32.013 \mathrm{~km}^{2}$, o rio apresenta uma extensão de 247km e abrange área de quatro estados: Bahia, Pernambuco, Sergipe e Alagoas, correspondendo à menor porção dentre as quatro subdivisões fisiográficas (MARTINS et al., 2011). Está situado no trecho entre a cidade de Paulo Afonso, Estado da Bahia e a sua foz, perfazendo um total de $210 \mathrm{~km}$ no sentido oeste-leste. Para efeito do presente estudo, aborda-se os perímetros irrigados Betume e Califórnia (Figura 1).

No estudo foram realizadas 22 campanhas de amostragem, mensalmente, de 03/2013 a 12/2014, com monitoramento do rio das Onça, rio Betume e drenagem do Betume, que são afluentes do baixo São Francisco, no estado de Sergipe. O rio da Onça, que está situado no extremo noroeste sergipano, no município de Canindé de São Francisco, possui uma área de drenagem de aproximadamente 108,6 km², recebendo água do perímetro irrigado Califórnia. O início das atividades do perímetro ocorreu no ano de 1987 e compondo uma área de 3.980 ha, com 333 lotes, incluindo os trabalhadores rurais que utilizam o sistema de irrigação artificial (bomba d'água), e os demais pelo sistema natural, que depende da chuva (SERGIPE, 2004). O monitoramento ocorreu nas coordenadas geográficas 24L 632344; 8930745.

Quanto ao perímetro irrigado Betume, tem uma área total de 6.698ha, com uma concentração de 764 produtores e situado à jusante de Neópolis abrange parte dos municípios de Ilha das Flores, Pacatuba e Neópolis. O monitoramento foi realizado nas coordenadas geográficas 24L 763227; 8848837 na margem do rio Betume. O perímetro irrigado Betume, possui 4 estações de bombeamento (EBs 01, 07, 08, 09), que recircular a água da rizicultura para o rio Betume ou rio São Francisco, por um canal de drenagem (que neste trabalho foi nomeado 'Drenagem do Betume'). O ponto de monitoramento neste local, foi nas coordenadas geográficas 24L 766368; 8847251.

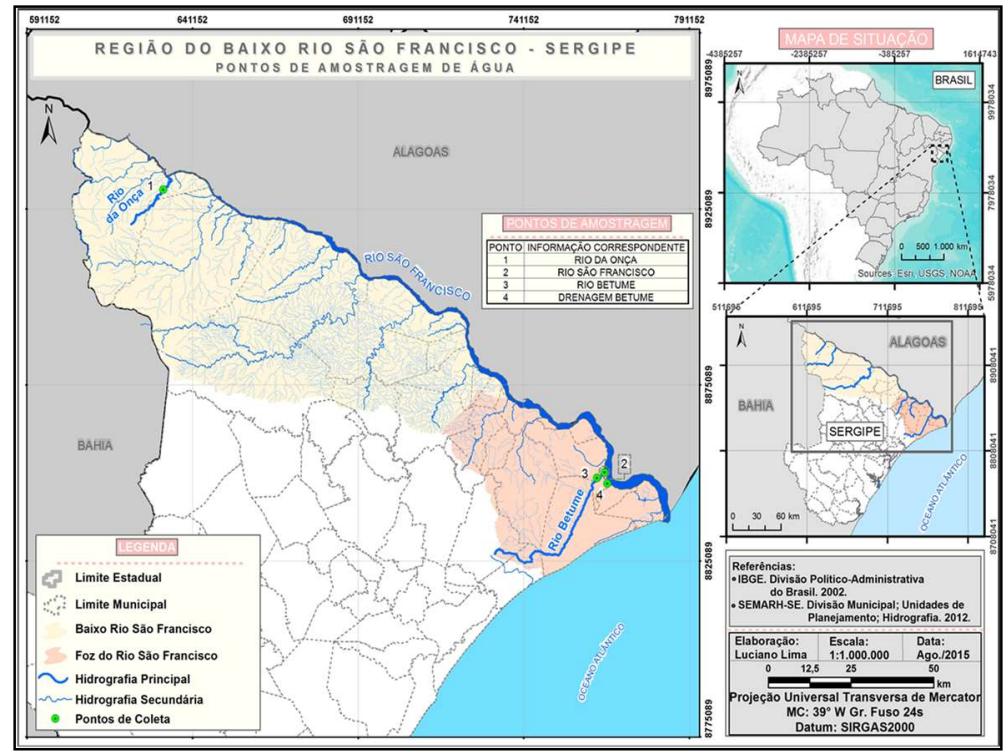

Figura 1: Localização do riacho da Onça em Canindé de São Francisco e do rio Betume em Ilha das Flores, Pacatuba e Neópolis, Sergipe. 
Com o objetivo de conhecer os impactos antrópicos na qualidade da água no baixo rio São Francisco, foram monitoradas as varáveis físico-químico da água: $\mathrm{pH}$, oxigênio dissolvido (OD), demanda química de oxigênio (DQO), demanda bioquímica de oxigênio (DBO), turbidez, sólidos totais dissolvidos (TDS), fósforo total, nitrato, $\mathrm{N}$-amoniacal, cor aparente, sólidos suspensos totais (SST), dureza total, fluoreto total, sulfatos, ferro total, manganês total, sódio, coliformes termotolerantes, temperatura, salinidade, cloreto e condutividade elétrica (Tabela 1 ).

Tabela 1: Resumo da metodologia analítica adotada.

\begin{tabular}{|c|c|c|c|}
\hline Parâmetros & Metodologia & $\mathrm{LQ}$ & Unidade \\
\hline $\mathrm{pH}$ & SMEWW, 2012, $4500 \mathrm{H}+\mathrm{B}$ & - & - \\
\hline OD & SMEWW, 2012, 4500-O C & - & $\mathrm{mg} \cdot \mathrm{O}_{2} \cdot \mathrm{L}^{-1}$ \\
\hline DBO & SMEWW, 2012, 5210-D & - & $\mathrm{mg} \cdot \mathrm{O}_{2} \cdot \mathrm{L}^{-1}$ \\
\hline $\mathrm{DQO}$ & SMEWW, 2012, 5220 D/Nova 60 Merck & - & $\mathrm{mg} \cdot \mathrm{O}_{2} \cdot \mathrm{L}^{-1}$ \\
\hline Turbidez & SMEWW, 2012, $2130 \mathrm{~B}$ & - & U.T \\
\hline TDS & SMEWW, 2012, $2510 \mathrm{~A}$ & - & $\mathrm{mg} \cdot \mathrm{L}^{-1}$ \\
\hline Fósforo total & SMEWW,4500-P E & 0,01 & mg.P.L.'-1 \\
\hline Nitrato & Cromatografia iônica (US EPA 300.0) & 0,011 & $\mathrm{mg} \cdot \mathrm{N}-\mathrm{NO}_{3} \cdot \mathrm{L}^{-1}$ \\
\hline $\mathrm{N}$-amoniacal & Cromatografia iônica (US EPA 300.0) & 0,039 & $\mathrm{mg} \cdot \mathrm{NH}^{3} \cdot \mathrm{L}^{-1}$ \\
\hline Cor aparente & SMEWW, 2012, $2120 \mathrm{C}$ & 0,2 & $\mathrm{uH}$ \\
\hline Sólidos suspensos Totais & SMEWW, 2012, 2540 D & - & mg. $\mathrm{L}^{-1}$ \\
\hline Dureza total & SMEWW, 2012, 2340 B (Cálculo) & - & $\mathrm{mg} \cdot \mathrm{CaCO}^{3} \cdot \mathrm{L}^{-1}$ \\
\hline Fluoreto total & Cromatografia iônica (US EPA 300.0) & 0,050 & $\mathrm{mg} \mathrm{F}^{-} \cdot \mathrm{L}^{-1}$ \\
\hline Sulfatos & Cromatografia iônica (US EPA 300.0) & 0,050 & $\mathrm{mg} \cdot \mathrm{SO}_{4} \cdot \mathrm{L}^{-1}$ \\
\hline Ferro total & AAS & 0,023 & mg.Fe. $L^{-1}$ \\
\hline Manganês total & AAS & 0,02 & mg. $\cdot \mathrm{Mn}^{2+} \cdot \mathrm{L}^{-1}$ \\
\hline Sódio & AAS & 0,044 & $\mathrm{mg} \cdot \mathrm{Na}^{+} . \mathrm{L}$ \\
\hline Colif. termotolerantes & SMEWW9221B & - & NMP. $100 \mathrm{~mL}^{-1}$ \\
\hline Temperatura & Sonda Multiparâmetros HANNA - HI9829 & - & ${ }^{\circ} \mathrm{C}$ \\
\hline Salinidade & Sonda Multiparâmetros HANNA - HI9829 & - & PSU \\
\hline Cloreto & SMEWW,4500-CL B & 2,42 & $\mathrm{mg} \cdot \mathrm{Cl}^{-} \cdot \mathrm{L}^{-1}$ \\
\hline Condutividade & Sonda Multiparâmetros HANNA - HI9829 & - & ? $\mathrm{S} . \mathrm{cm}^{-1}$ \\
\hline
\end{tabular}

Fonte: Laboratório ITPS e LQA. Sonda Multiparâmetros. Standard Methods for the Examination of Water and Wastewater (SMEWW).

Os parâmetros foram correlacionados por meio do software SPSS 13.0 (Statistical Package for the Social Sciences) utilizando os dados físico-químicos do rio da Onça, rio Betume, rio São Francisco e a drenagem do perímetro irrigado Betume, com o objetivo de identificar as inter-relações entre um grande número de parâmetros e explicar em termos de suas dimensões que influências pode haver nas variações quantitativas de suas varáveis.

Diante disso, foi necessário determinar o grau de dispersão entre os parâmetros de qualidade da água. Para isto, os dados foram compilados conforme o grau dispersão (Tabela 2), de forma que, os parâmetros com maior correlação, poderão apresentar resultados mais próximos da realidade ambiental da região de estudo.

Tabela 2: Critério de avaliação do grau de dispersão.

\begin{tabular}{|l|l|}
\hline Valor do Coeficiente de Variação (CV) & Grau de Dispersão \\
\hline$<0,50$ & Baixo \\
\hline 0,50 a 1,00 & Médio \\
\hline 1,00 a 1,20 & Alto \\
\hline$>1,20$ & Elevado \\
\hline
\end{tabular}

Fonte: Adaptado de França (2009). 
A análise multivariada consiste num conjunto de métodos estatísticos que utiliza, simultaneamente, todas os parâmetros na interpretação teórica do conjunto de dados obtidos (COSTELLO et al., 2005). A análise de dados multivariados conta com diversas técnicas, dentre elas, pode-se citar: análise fatorial, regressão múltipla e correlação múltipla, análise discriminante múltipla, análise multivariada de variância e covariância, análise conjunta, correlação canônica, análise de agrupamentos e escalonamento.

A utilização de análise multivariada, por meio de análise fatorial vem sendo amplamente utilizada para entender o melhor comportamento dos parâmetros de qualidade da água (GIRÃO et al., 2007; KUMARESAN et al., 2008; SCHAGERL et al., 2010; SOJKA et al., 2008; SONG et al., 2011; YEUNG, 1999).

A análise fatorial inclui a análise de componentes principais e análise dos fatores comuns. É aplicada quando há um número grande de parâmetros e correlacionadas entre si, com o objetivo de identificar um número menor de novos parâmetros alternativas, não correlacionadas e que, de algum modo, sumarizem as informações principais dos parâmetros originais encontrando os fatores ou parâmetros latentes (MINGOTI, 2005).

A matriz de correlação de Pearson é o primeiro passo para a aplicação da análise de componentes principais/análise de fator comum, ou seja, a transformação da matriz de dados em uma matriz de correlação, a qual permitirá a seleção dos parâmetros mais significativas, ou seja, aquelas que apresentarem coeficientes de correlação maiores que 0,5 (WANG et al., 2011). Em seguida, torna-se necessário certificarse que os dados são consistentes com a estrutura da análise fatorial para isso se utiliza o teste de esfericidade de Bartlett e a medida de adequacidade da amostra de Kaiser-Meyer-Olkin (KMO) que são testes empregados para verificar a validade do emprego da Análise Fatorial.

Em seguida, foi utilizado a representação gráfica do tipo box plot, das concentrações dos parâmetros de qualidade da água. Esta metodologia possibilita a visualização das grandezas estatísticas como média, mediana, máximo, mínimo, $1^{\circ}$ quartil, $3^{\circ}$ quartil e os valores extremos. $01^{\circ}$ quartil (inferior) representa um percentil de $25 \%$ e o $3^{\circ}$ quartil (superior) que representa um percentil de $75 \%$ dos dados observados. Além disso, possibilita visualizar as variâncias destas varáveis e suas influências ambientais. Os pontos extremos podem indicar erros de amostragem, de medida e, mesmo, de transcrição dos dados, ou seja, um comportamento fora do habitual, ainda assim, está representação gráfica está sendo utilizadas por diversos autores (RAMOS JUNIOR et al., 2012; RODRIGUES et al., 2014; THEBALDI et al., 2011).

\section{RESULTADOS E DISCUSSÃO}

As campanhas de monitoramento foram realizadas em três mananciais em diferentes regiões tornando complexo a inter-relação dos parâmetros físico-químicos no baixo São Francisco. Com os dados foi realizada uma análise do grau de dispersão entre as varáveis, comparando-as entre si e determinando valores possíveis de serem analisados em virtude das diferentes características de solo e sazonais das regiões (Tabela 3). Esta comparação foi originalmente proposta por França (2009), utilizando a relação de desvio padrão dividido pela média e assim comparar a variação de conjuntos de observações e classificar o grau de dispersão dos parâmetros. 
Tabela 3: Estatística descritiva dos 22 parâmetros.

\begin{tabular}{|l|l|l|l|l|l|}
\hline Parâmetros & Min. & Máx. & Média & Desvio Padrão & Coef. de Variação \\
\hline Manganês Total & 27,0 & 0,01 & 0,88 & 4,62 & 5,26 \\
\hline Fluoreto Total & 65,10 & 0,03 & 2,16 & 10,13 & 4,68 \\
\hline Coliformes Termotolerantes & $17 \times 10^{4}$ & 1,80 & 14.374 & 36.713 & 2,55 \\
\hline Ferro Total & 4,54 & 0,00 & 0,93 & 1,40 & 1,50 \\
\hline Sódio & 300,87 & 1,56 & 46,51 & 65,43 & 1,40 \\
\hline Nitrogênio - Nitrato & 0,28 & 0,09 & 0,80 & 1,12 & 1,39 \\
\hline Cor Aparente & 433,0 & 0,20 & 72,44 & 99,86 & 1,37 \\
\hline Sulfatos (SULF) & 115,0 & 0,05 & 27,43 & 32,87 & 1,19 \\
\hline Cloretos (CL) & 300,50 & 2,55 & 75,46 & 90,31 & 1,19 \\
\hline Turbidez (TURB) & 146,0 & 1,20 & 31,39 & 37,25 & 1,18 \\
\hline Fósforo Total (Ptotal) & 0,45 & 0,01 & 0,08 & 0,10 & 1,18 \\
\hline Salinidade (SAL) & 0,88 & 0,04 & 0,19 & 0,23 & 1,16 \\
\hline Condutividade (CE) & 1752 & 58,64 & 297,95 & 336,55 & 1,13 \\
\hline Sólidos Totais Dissolvido (TDS) & 931,0 & 25,04 & 195,34 & 215,32 & 1,10 \\
\hline Nitrogênio - Amoniacal (NH3) & 1,12 & 0,05 & 0,19 & 0,19 & 1,028 \\
\hline Sólidos Suspensos Totais (SST) & 121,0 & 1,00 & 35,56 & 35,12 & 0,98 \\
\hline Dureza Total (DT) & 188,0 & 0,09 & 48,01 & 47,08 & 0,98 \\
\hline Demanda Bioquímica de Oxigênio (DBO) & 112,57 & 1,36 & 27,69 & 23,25 & 0,84 \\
\hline Demanda Química de Oxigênio(DQO) & 261,08 & 4,30 & 75,17 & 56,86 & 0,76 \\
\hline Oxigênio Dissolvido (OD) & 9,10 & 0,07 & 4,33 & 2,02 & 0,47 \\
\hline Potencial Hidrogeniônico (pH) & 5,40 & 9,08 & 7,21 & 0,83 & 0,11 \\
\hline Temperatura (TEMP) & 31,0 & 23,30 & 27,41 & 1,55 & 0,05 \\
\hline
\end{tabular}

Os dados da Tabela 3 foram comparados com os dados da Tabela 2, para classificação do grau de dispersão. Sendo assim os valores acima de 1,2 apresentaram alto grau de dispersão, isto pode ser explicado em razão destes parâmetros não serem estáveis, ou seja, a alta dispersão dos valores durante a análise dos resultados no monitoramento dos rios pode dificultar a interpretação dos dados estatisticamente.

$\mathrm{Na}$ tabela 4 foi gerada a matriz de correlação composta dos parâmetros estudadas para a bacia hidrográfica do rio São Francisco. A análise de componentes principais resultou na redução de um dos parâmetros analisadas. Neste caso, o Nitrogênio-amoniacal (NH3), não se mostrou significativa na explicação da variância total, e nem obteve correlação com as outras varáveis, podendo ser desconsiderada neste estudo estatístico. Neste tipo de análise é interessante diminuir o espaço amostral uma vez que, reduzindo o número de parâmetros de qualidade de água, reduz-se também o número de análises laboratoriais, economizando tempo e recurso financeiro (ZENG et al., 2005).

Tabela 4: Matriz de correlação dos parâmetros originais de qualidade da água no rio São Francisco, o riacho da Onça, o rio Betume e a Drenagem do Betume.

\begin{tabular}{|l|l|l|l|l|l|l|l|l|l|l|l|l|l|l|}
\hline & $\mathrm{pH}$ & $\mathrm{CE}$ & $\mathrm{OD}$ & $\mathrm{P}_{\text {total }}$ & TDS & DQO & DBO & SST & TUR & $\mathrm{CL}$ & $\mathrm{DT}$ & SUL & TEM & SAL \\
\hline $\mathrm{pH}$ & 1,00 & & & & & & & & & & & & & \\
\hline CE & 0,48 & 1,00 & & & & & & & & & & & & \\
\hline OD & $\mathbf{0 , 6 6}$ & 0,27 & 1,00 & & & & & & & & & & & \\
\hline $\mathrm{P}_{\text {total }}$ & $\mathbf{0 , 5 1}$ & $\mathbf{0 , 8 0}$ & 0,20 & 1,00 & & & & & & & & & & \\
\hline TDS & $\mathbf{0 , 6 6}$ & $\mathbf{0 , 7 9}$ & 0,22 & $\mathbf{0 , 7 5}$ & 1,00 & & & & & & & & & \\
\hline DQO & $-0,06$ & $-0,03$ & 0,06 & $-0,15$ & $-0,01$ & 1,00 & & & & & & & & \\
\hline DBO & 0,03 & $-0,14$ & 0,05 & $-0,14$ & $-0,02$ & $\mathbf{0 , 5 4}$ & 1,00 & & & & & & & \\
\hline SST & $-0,47$ & 0,00 & $-0,42$ & 0,08 & $-0,15$ & 0,08 & $-0,06$ & 1,00 & & & & & & \\
\hline TUR & $-0,35$ & 0,09 & $-0,36$ & 0,00 & $-0,05$ & 0,17 & $-0,02$ & $\mathbf{0 , 6 5}$ & 1,00 & & & & & \\
\hline CL & $\mathbf{0 , 5 9}$ & $\mathbf{0 , 6 6}$ & 0,26 & $\mathbf{0 , 6 3}$ & $\mathbf{0 , 8 2}$ & 0,10 & $-0,10$ & $-0,15$ & $-0,06$ & 1,00 & & & & \\
\hline DT & $\mathbf{0 , 6 9}$ & $\mathbf{0 , 7 6}$ & 0,28 & $\mathbf{0 , 7 6}$ & $\mathbf{0 , 8 5}$ & $-0,12$ & $-0,06$ & $-0,18$ & $-0,12$ & $\mathbf{0 , 7 8}$ & 1,00 & & & \\
\hline SUL & $\mathbf{0 , 7 0}$ & $\mathbf{0 , 6 8}$ & 0,31 & $\mathbf{0 , 7 4}$ & $\mathbf{0 , 8 5}$ & $-0,09$ & $-0,02$ & $-0,19$ & $-0,13$ & $\mathbf{0 , 8 3}$ & $\mathbf{0 , 9 0}$ & 1,00 & & \\
\hline TEM & $-0,35$ & $-0,09$ & $-0,42$ & $-0,05$ & $-0,03$ & 0,10 & 0,17 & $\mathbf{0 , 5 2}$ & 0,40 & $-0,14$ & $-0,20$ & $-0,16$ & 1,00 & \\
\hline SAL & 0,49 & $\mathbf{0 , 8 7}$ & 0,23 & $\mathbf{0 , 8 1}$ & $\mathbf{0 , 7 8}$ & $-0,14$ & $-0,21$ & $-0,02$ & 0,02 & $\mathbf{0 , 7 3}$ & $\mathbf{0 , 7 9}$ & $\mathbf{0 , 7 1}$ & $-0,23$ & 1,00 \\
\hline
\end{tabular}


Os parâmetros que apresentaram maiores coeficientes de correlação entre si foram $\mathrm{pH}$, condutividade elétrica, fósforo total, TDS, DQO, SST, cloreto, sulfato, dureza total e salinidade. Pode-se observar, também, que a relação entre sulfato e dureza total foi 0,9 apresentando correlação muito forte e positiva. Sabe-se que a presença de sulfato na água doce, em sistemas públicos de abastecimento, em concentração elevadas, atua como laxante e associado a íons de cálcio e magnésio promove dureza permanente na água podendo ser um indicativo de poluição em fase de decomposição de matéria orgânica (RICHTER, 2009).

Dando continuidade, tem-se o critério de Kaiser-Meyer-Olkin (KMO), em que o valor encontrado foi igual a 0,804, sendo está acima de 0,5, e é considerado mais adequados para aplicação da análise fatorial, portanto foram definidos 14 parâmetros conforme a Tabela 5.

Para uma melhor interpretação destes dados foi aplicada a rotação ortogonal dos fatores pelo método Varimax (Tabela 5). Esse processo maximiza a variância entre os fatores alterando a raiz característica sem afetar a proporção da variância total explicada pelo conjunto, como também minimiza a ocorrência de uma variável possuir altas cargas fatoriais para diferentes fatores, permitindo que uma variável seja facilmente identificada com um único fator (ANDRADE et al., 2007; CORRAR et al., 2007).

Tabela 5: Matriz das cargas de fatores comuns rotacionada pelo método Varimax.

\begin{tabular}{|l|l|l|l|}
\hline \multirow{2}{*}{ Parâmetros } & Componente & Componente & Componente \\
\cline { 2 - 4 } & 3 & 3 & 3 \\
\hline TDS & 0,927 & $-0,081$ &, 060 \\
\hline DT & 0,914 & $-0,188$ & $-0,044$ \\
\hline SUL & 0,894 & $-0,205$ & 0,023 \\
\hline SAL & 0,892 & $-0,023$ & $-0,195$ \\
\hline CE & 0,887 & 0,037 & $-0,069$ \\
\hline Ptotal & 0,874 & 0,051 & $-0,150$ \\
\hline CL & 0,857 & $-0,129$ & 0,083 \\
\hline pH & 0,545 & $-0,597$ & 0,095 \\
\hline OD & 0,263 & $-0,674$ & 0,156 \\
\hline TURB & 0,055 & 0,795 & 0,077 \\
\hline SST & $-0,010$ & 0,865 & $-0,033$ \\
\hline TEMP & $-0,065$ & 0,705 & 0,210 \\
\hline DQO & $-0,027$ & 0,108 & 0,858 \\
\hline DBO & $-0,085$ & $-0,036$ & 0,863 \\
\hline Autovalor & 6,401 & 2,494 & 1,627 \\
\hline \% variância explicada & 45,723 & 17,817 & 11,625 \\
\hline \% variância acumulada & 45,723 & 63,540 & 75,164 \\
\hline
\end{tabular}

Para sintetizar as informações obtidas para a matriz de componente rotativa 1, 2 e 3 (CP1, CP2 e CP3), utilizou-se a representação gráfica dos parâmetros. Observa-se na Figura 2, a formação dos grupos de parâmetros para CP1: TDS, DT, SUL, SAL, CE e CL no lado direito do gráfico com conteúdo mineral. Na CP2, os parâmetros ficaram agrupadas no lado superior foram SST, TURB e TEMP na parte inferior OD e PH demonstrando a relação inversamente proporcional, com parâmetros químicos. A CP3 está DQO e DBO, com matéria orgânica dissolvida.

O parâmetro sólido total dissolvido teve grande contribuição na condutividade elétrica que se relaciona com salinidade e dureza (KUMARESAN et al., 2008). Esses parâmetros podem estar sendo influenciados provavelmente pela lixiviação intensa dos sais acumulados no solo, principalmente no riacho 
da Onça, em virtude das atividades agrícolas no perímetro irrigado Califórnia (RESENDE et al., 2014).

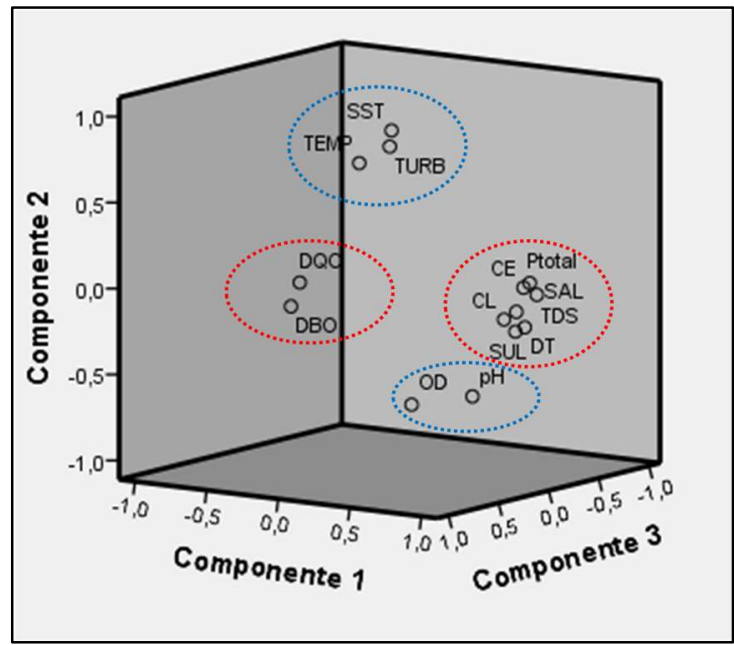

Figura 2: Cargas dos três fatores com rotação ortogonal pelo método Varimax.

O pH e OD apresentaram valores negativos, em relação ao CP2. Richter (2009) cita que águas com elevadas concentrações na cor, turbidez e TDS, aumentam os custos de tratamento, diminui a vida útil dos filtros nas estações de tratamento, fazendo subir os valores pagos pelos consumidores em geral. Para avaliar as concentrações dos parâmetros químicos e sua influência no rio São Francisco foi utilizado séries temporais e diagramas de caixa (Box plot) com intuito de auxiliar a interpretação dos dados (Figura 3). Dentre eles temse a condutividade elétrica, que demonstrou valores elevados no riacho das Onça, rio Betume e drenagem do Betume (Figura 3A), considerando que a CE reflete a concentração de íons em solução (WETZEL et al., 2001). Valores similares foram encontrados para a condutividade elétrica no riacho Jacaré em Sergipe, mas ainda não apresenta risco de salinização do solo (LUCAS et al., 2014).

Provavelmente no riacho da Onça está concentração de sais solúveis na água, pode estar relacionado ao nível fluviométrico ser mais baixo nesta bacia hidrográfica. Valores similares aos observados neste estudo foram relatados no rio dos Sinos, Rio Grande do Sul, que foi caracterizado como de água com baixa qualidade e fortes atividades antrópicas naquele local (BLUME et al., 2010).

Os altos valores encontrados para os sólidos totais dissolvidos (TDS) na água do riacho da Onça (Figura 3B), pode estar relacionado a drenagem superficial de áreas rurais que apresentam como possível efeito poluidor a salinidade. Os valores de condutividade elétrica E sólidos totais dissolvidos (TDS), apresentaram relação linear, com altos coeficientes de correlação durante monitoramento sazonal da qualidade da água, no córrego água limpa em Minas Gerais (PIERON et al., 2015).

O excesso de sais em solos do perímetro irrigado Califórnia pode ser uma consequência do manejo inadequado e uso exagerado de água na irrigação. Sendo assim o assoreamento dos drenos naturais nos talvegues naturais, promovem o escoamento do excesso de água da irrigação e das chuvas e está contribuindo para os altos valores de salinidade detectados no riacho da Onça (Figura 3C). Isso revela a necessidade de mudança de comportamento dos agricultores quanto ao manejo da irrigação e do solo (AGUIAR NETTO et al., 2007) 


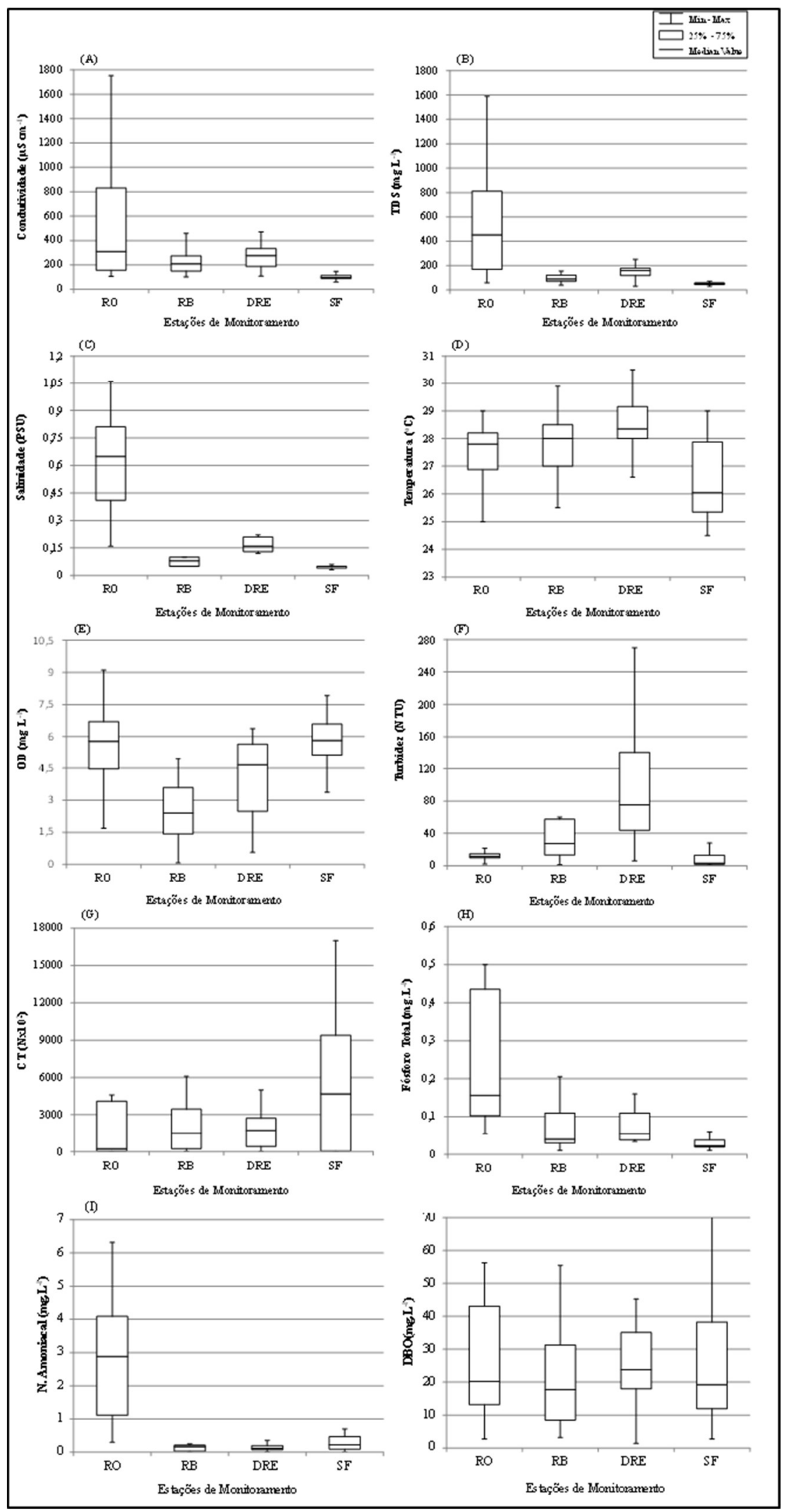

Figura 3: Distribuição por Box Plot dos Parâmetro de Qualidade da água no Riacho da Onça (RO), rio Betume (RB), Drenagem do Betume (DRE) e rio São Francisco (SF), Sergipe.

A ausência de áreas de proteção permanente, como também os despejos do esgoto doméstico nas regiões de estudo podem estar contribuindo para o aumento da temperatura da água (Figura 3D), e diminuindo a concentração de OD (Figura 3E), nos afluentes do rio São Francisco. A eutrofização provoca uma coloração turva nos corpos d'água e reduz os valores de oxigênio dissolvido. Com o aumento da 
concentração de nutrientes o crescimento e a multiplicação do fitoplâncton (Algas), favorece o aumento da turbidez da água, que pode ser observado na drenagem do Betume (Figura 3F).

As bactérias coliformes termotolerantes são indicadoras de poluição por esgotos domésticos, sendo assim essa evidência se torna realidade nas margens do rio São Francisco, com a presença de bares e prainha que são utilizados pelos moradores das cidades ribeirinhas, contribuindo para os índices elevados de coliforme termotolerante (Figura 3G). Na região hidrográfica do rio Pardo - RS, os principais problemas ambientais detectados dizem respeito à contaminação orgânica e eutrofização da água, basicamente em função dos parâmetros fosfato, coliformes termotolerantes e turbidez (MORETTO et al., 2012). Sua presença indica risco da ocorrência de outros microrganismos patogênicos, responsáveis pela transmissão de doenças de veiculação hídrica, tais como febre tifoide, febre paratifoide, desinteria bacilar e cólera.

O fósforo, como parâmetro da qualidade da água, não é considerado tóxico, mas contribui para acelerar os processos de eutrofização, sendo assim foi constatado índices elevado no riacho da Onça, influenciado pelas atividades rurais do perímetro irrigado (Figura $3 \mathrm{H}$ ). Quando este encontra-se associado a compostos de nitrogênio, em grandes quantidades nos corpos d'água (Figura 3I), causa um crescimento excessivo das algas, o que pode prejudicar o abastecimento público, a recreação e a preservação da vida aquática (LOBO et al., 2010).

A presença de áreas agrícolas, e a drenagem da água do perímetro irrigado Califórnia por fontes difusas, para o riacho da Onça, evidencia o elevado índice de fósforo e nitrogênio, durante o período de monitoramento. Segundo Vasco et al. (2011), na bacia hidrográfica do rio Poxim as concentrações de fósforo cresceram no período chuvoso, possivelmente pela a drenagem de áreas agrícolas nesta região.

A presença da matéria orgânica e os elevados valores de DBO confirmam o processo de esgotamento do oxigênio na água, que poderá provocar o desaparecimento de peixes e outras formas de vida aquática (Figura 3J). Fica evidenciado através dos parâmetros analisados neste estudo, que há um processo de degradação, tanto dos afluentes, como do rio São Francisco, provocado pela expansão das atividades agrícolas e o esgoto doméstico que margeiam os rios de estudo.

Vale salientar que este monitoramento vem ressaltar as condições de vida da maioria destes produtores dos Perímetros irrigados, que não possuem água potável e nem esgoto sanitário, e utiliza a água bombeada do rio São Francisco para irrigação, em suas residências e sem o tratamento adequado. A falta de assistência técnica, a baixa produtividade, a exploração dos atravessadores na venda de seus produtos, a falta de apoio das prefeituras, corroborando para o desinteresse e o empobrecimento dos irrigantes na sustentabilidade ambiental e econômica da região.

\section{CONCLUSÕES}

Aplicação de ferramenta estatística combinadas com os dados de monitoramento possibilita o pesquisador identificar suas correlações, e avaliar as suas relações com os parâmetros de qualidade da água no meio ambiente. A aplicação da análise fatorial ao conjunto de parâmetros resultou na seleção de três fatores que representam os parâmetros físico-químico da qualidade da água dos rios em estudo. A rotação 
ortogonal Varimax dos fatores mostrou que os parâmetros da qualidade da água estão relacionados principalmente com o conteúdo mineral, nutricional, matéria orgânica e grau de poluição.

O perímetro irrigado Califórnia é uma fonte potencial de contaminação da qualidade da água do riacho da onça, principalmente quando se observa os elevados valores de salinidade, $O D$, fósforo, nitrogênio e DBO, e assim comprometer a sustentabilidade deste manancial. No perímetro irrigado Betume percebe-se que o manejo da cultura do arroz está provocando a eutrofização, em virtude do aporte de fertilizantes como nitrogênio e fósforo, que possibilita o crescimento mais intenso de seres vivos que utilizam estes nutrientes, especialmente as algas, prejudicando seriamente o abastecimento público e a irrigação.

As baixas concentrações de oxigênio dissolvido e as altas temperaturas dos mananciais do rio Betume e drenagem do Betume, está relacionado a ausência de áreas de proteção permanente, como também os despejos do esgoto doméstico nas regiões de estudo do Baixo São Francisco. O uso de modelos estatísticos para analisar os resultados dos parâmetros físico-químicos, pode demonstrar a problemática nos perímetros irrigados Betume e Califórnia no Baixo São Francisco quanto ao manejo e qualidade da água dos mananciais.

\section{REFERÊNCIAS}

AGUIAR NETTO, A. O. A.; GOMES, C. C. S.; LINS, C. C. V.; BARROS, A. C.; CAMPECHE, L. F. S. M.; BANCO, F. F.. Características químicas e salino-sodicidade dos solos do Perímetro Irrigado Califórnia, SE, Brasil. Ciência Rural, v.37, n.6, 2007. DOI: http://dx.doi.org/10.1590/S010384782007000600021

ANDRADE, E. M.; ARAÚJO, L. F. P.; ROSA, M. F.; GOMES,R.B.; LOBATO, F. A. O.. Assessment of the surface water quality in the upland of Acaraú watershed, Ceará, Brazil. Revista Ciência Rural, v. 37 n.6, p.1791-1797. 2007. DOI: http://dx.doi.org/10.1590/S0103-84782007000600045

BARROS, A. C.; AGUIAR NETTO, A. O.; SANTA ROSA, Y. R.; MELO, A. S.; BRITO, M. C. B. Qualidade da Irrigação por Microaspersao em Pomar de Banana (Musa spp.) fertirrigado. Revista Magistra, Cruz das Almas, v.20, n.2, p.197-203, 2008.

BLUME, K. K.; MACEDO, J. C.; MENEGUZZI, A.; SILVA, L. B.; QUEVEDO, D. M.; RODRIGUES, M. A. S.. Water quality assessment of the Sinos River, Southern Brazil. Brazilian Journal of Biology, v.70, n.4, p.1185-1193. DOI: http://dx.doi.org/10.1590/1519-6984.01613suppl

CORRAR, L. J.; DIAS FILHO, J. M.; PAULO, E.. Análise Multivariada: para os cursos de administração, ciências contábeis e economia. São Paulo: Atlas, 2007.

COSTELLO, A. B; OSBORNE, J. W.. Best practices in exploratory factor analysis: Four recommendations for getting the most from your analysis. Practical Assessment Research \& Evaluation, v.10, n.7, p.13-24, 2005.

FERREIRA, R. A.; SILVA-MANN, R.; ARAGÃO, A. G.; REZENDE, A. M. S.; SANTOS, T. I. S.; SANTOS, P. L.; CARVALHO, S. V. A.. As Áreas Ciliares Na Região Do Baixo Rio São Francisco: Processo De Ocupação E Sua Recuperação. São Cristóvão: UFS, 2011.
FRANÇA, M. S.. Análise Estatística Multivariada dos Dados de Monitoramento de Qualidade de Água da Bacia do Alto Iguaçu: Uma Ferramenta para a Gestão de Recursos Hídricos. Dissertação (Mestrado em Recursos Hídricos e Ambiental) - Universidade Federal do Paraná, Curitiba, 2009.

GIRÃO, E. G.; ANDRADE, E. M.; ROSA, M. F.; ARAÚJO, L. F. P.; MEIRELES, C. M.. Water quality assessment of the Jaibaras River, Ceará, Brazil using principal component analysis. Revista Ciência Agronômica, v.38 n.1, p.17-24, 2007.

HOLANDA, F. S. R.; ROCHA, I. P.; OLIVEIRA, V. S. Estabilização de taludes marginais com técnicas de bioengenharia de solos no Baixo São Francisco. Revista Brasileira de Engenharia Agrícola e Ambiental, v.12, n.6, p.570-575, 2008. DOI http://dx.doi.org/10.1590/S141543662008000600002

KUMARESAN, M.; RIYAZUDDIN, P.. Factor analysis and linear regression model (LRM) of metal speciation and physicochemical characters of groundwater samples. Environmental Monitoring and Assessment, v.138, p.65-79, 2008. DOI: http://dx.doi.org/10.1007/s10661-007-9761-8

LOBO, E. A.; WETZEL, C. E.; ECTOR, L.; KATOH. K.; BLANCO, S.; MAYAMA, S.. Response of epilithic diatom community to environmental gradients in subtropical temperate Brazilian rivers. Limnetica, Madrid, v.29, n.2, p.323-34, 2010. DOI: http://dx.doi.org/10.23818/limn.29.27

LUCAS, A. A. T.; MOURA, A. S. A.; AGUIAR NETTO, A.; O, FACCIOLI, G. G.; SOUSA, O. F. Qualidade da Água no Riacho Jacaré, Sergipe Brasil usada para Irrigação. Revista Brasileira de Agricultura Irrigada, v.8, n.2, p.98-105, 2014. DOI: http://dx.doi.org/10.7127/rbai.v8n200228

MARTINS, D. M. F.; CHAGAS, R. M.; MELO NETO, J. O.; MÉLLO JÚNIOR, A. V.. Impactos da construção da usina hidrelétrica de Sobradinho no regime de vazões no Baixo São Francisco. Revista Brasileira de Engenharia Agrícola e 
Ambiental, v.15, n.9, p.1054-1061, 2011. DOI: http://dx.doi.org/10.1590/S1415-43662011001000010

MINGOTI, S. A.. Análise de dados através de métodos de estatística multivariada: uma abordagem aplicada. Belo Horizonte: UFMG, 2005.

MORETTO, D. L.; PANTA, R. E.; COSTA, A. B.; LOBO, E. A. Calibration of water quality index (WQI) based on Resolution no 357/2005 of the Environment National Council (CONAMA). Acta Limnológica Brasiliensia, v.24, p.29-42, 2012. DOI: http://dx.doi.org/10.1590/S2179975X2012005000024

PIERON, J. P.; BRANCO, K. G. R.; INACHVILI, I.; FERREIRA, G C.. Monitoramento Sazonal da Qualidade da Água, na Subbacia Hidrográfica do Córrego Água Limpa, em seu trecho afetado pela Mineração de Níquel, no município de Pratápolis, Minas Gerais. Geociências, São Paulo, v.34, p.402-410, 2015

RAMOS JUNIOR, A. B. S.; CRUZ, M. J. M.. Variabilidade Espaço-Temporal de Parâmetro Físico-Químico e Metais Pesados no Rio São Paulo, Município de candeias, Bahia. Revista Geociências, São Paulo, v.31, n.4, p.622-637, 2012.

RESENDE, R. S.; AMORIM, J. R. A.; CRUZ, M. A. S.; MENESES, T. N.. Distribuição espacial e lixiviação natural de sais em solos do Perímetro Irrigado Califórnia, em Sergipe. Revista Brasileira Engenharia Agrícola Ambiental, v.18, 2014, p.4652, 2014. DOI: http://dx.doi.org/10.1590/18071929/agriambi.v18nsupps46-s52

RICHTER, C. A.. Água: métodos e tecnologia de tratamento. São Paulo: Edgard Blücher, 2009.

RODRIGUES, E. R. D.; HOLANDA, I. B. B.; CARVALHO, D. P.; BERNARDI, J. V. E.; MANZATTO, A. G.; BASTOS, W. B. Distribuição espacial da qualidade da água subterrânea na área urbana da cidade de Porto Velho, Rondônia. Revista Scientia Amazônia, v.3, n.3, p.97-105, 2014.

SCHAGERL, M.; BLOCH, I.; ANGELER, D. G.; FESL, C.. The use of urban clay-pit ponds for human recreation: assessment of impacts on water quality and phytoplankton assemblages. Environmental Monitoring and Assessment, v.165, p.283293, 2010. DOI: http://dx.doi.org/10.1007/s10661-0090945-2

SERGIPE. Programa de Desenvolvimento Sustentável do Semiárido Sergipano. Documento Conceptual do Projeto. Aracaju: Governo do Estado de Sergipe, 2004.
SOJKA, M.; SIEPAK, M.; ZIOŁA, A.; FRANKOWSKI, M.; MURATBŁAZEJEWSKA, S.; SIEPAK, J.. Application of multivariate statistical techniques to evaluation of water quality in the Mała Wełna River (Western Poland). Environmental Monitoring and Assessment, v.147, n.1-3, p.159-170, 2008. DOI: http://dx.doi.org/10.1007/s10661-007-0107-3

SONG, M. W.; HUANG, P.; LI, F.; ZHANG, H.; XIE, K. Z.; WANG, X. H.; HE, G. X.. Water quality of a tributary of the Pearl River, the Beijiang, Southern China: implications from multivariate statistical analyses. Environmental Monitoring and Assessment, v.172, p.589-603, 2011. DOI: http://dx.doi.org/10.1007/s10661-010-1358-y

THEBALDI, M. S.; SANDRI, S.; FELISBERTO, A. B.; ROCHA, M. S; NETO, S. A.. Qualidade da água de um córrego sob influência de efluente tratado de abate bovino, Revista Brasileira de Engenharia Agrícola e Ambiental, v.15, n.3, p.302-309, 2011. DOI: http://dx.doi.org/10.1590/S141543662011000300012

TRINDADE, A. L. C.; ALMEIDA, K. C. B.; BARBOSA, P. E.; OLIVEIRA, S. M. A. C.. Tendências temporais e espaciais da qualidade das águas superficiais da sub-bacia do Rio das Velhas, estado de Minas Gerais. Eng. Sanit. Ambient., Rio de Janeiro, v.22, n.1, p.13-24, 2017. DOI: http://dx.doi.org/10.1590/s1413-41522016131457

VASCO, A. N.; BRITTO, F. B.; PEREIRA, A. P. S.; MELLO JUNIOR, A. V.; GARCIA, C. A. B.; NOGUEIRA, L. C.. Avaliação espacial e temporal da qualidade da água na sub-bacia do rio Poxim, Sergipe, Brasil. Revista Ambi-Agua, v.6, n.1, p.118130, 2011. DOI: http://dx.doi.org/10.4136/ambi-agua.178

WANG, L.; WANG, Y.; XU, C.; AN, Z.; WANG, S.. Analysis and evaluation of the source of heavy metals in water of the River Changjiang. Environmental Monitoring and Assessment, v.173, p.301-313, 2011. DOI: http://dx.doi.org/10.1007/s10661-010-1388-5

WETZEL, R. G.; LIKENS, G. E.. Limnological analyses. New York: Springer-Verlag, 2001.

YEUNG, I. M. H.. Multivariate analysis of the Hong Kong Victoria Harbour water quality data. Environmental Monitoring and Assessment, n.59, p.331-342, 1999. DOI: http://dx.doi.org/10.1023/A:1006177824327

ZENG, X.; RASMUSSEN, T. C.. Multivariate statistical characterization of water quality in Lake Lanier, Georgia, USA. Journal Environmental Quality, v.34, p.1980-1991. 2005. DOI: http://dx.doi.org/10.2134/jeq2004.0337

A CBPC - Companhia Brasileira de Produção Científica (CNPJ: 11.221.422/0001-03) detém os direitos materiais desta publicação. Os direitos referem-se à publicação do trabalho em qualquer parte do mundo, incluindo os direitos às renovações, expansões e disseminações da contribuição, bem como outros direitos subsidiários. Todos os trabalhos publicados eletronicamente poderão posteriormente ser publicados em coletâneas impressas sob coordenação da Sustenere Publishing, da Companhia Brasileira de Produção Científica e seus parceiros autorizados. Os (as) autores (as) preservam os direitos autorais, mas não têm permissão para a publicação da contribuição em outro meio, impresso ou digital, em português ou em tradução. 